\title{
Pten Deletion Promotes Regrowth of Corticospinal Tract Axons 1 Year after Spinal Cord Injury
}

\author{
Kaimeng Du, ${ }^{1,2 *} \mathbb{C}$ Susu Zheng, ${ }^{1,2 \star}$ Qian Zhang, ${ }^{1,2 *}$ Songshan Li, ${ }^{1,2}$ Xin Gao, ${ }^{1,2}$ Juan Wang, ${ }^{3}$ Liwen Jiang, ${ }^{3}$ and Kai Liu ${ }^{1,2}$ \\ ${ }^{1}$ Division of Life Science, State Key Laboratory of Molecular Neuroscience, and ${ }^{2}$ Center of Systems Biology and Human Health, School of Science and \\ Institute for Advance Study, The Hong Kong University of Science and Technology, Clear Water Bay, Kowloon, Hong Kong, People's Republic of China, and \\ ${ }^{3}$ School of Life Sciences, Centre for Cell and Developmental Biology and State Key Laboratory of Agrobiotechnology, The Chinese University of Hong Kong, \\ Shatin, New Territories, Hong Kong, People's Republic of China
}

Chronic spinal cord injury (SCI) is a formidable hurdle that prevents a large number of injured axons from crossing the lesion, particularly the corticospinal tract (CST). This study shows that Pten deletion in the adult mouse cortex enhances compensatory sprouting of uninjured CST axons. Furthermore, forced upregulation of mammalian target of rapamycin (mTOR) initiated either 1 month or 1 year after injury promoted regeneration of CST axons. Our results indicate that both developmental and injury-induced mTOR downregulation in corticospinal motor neurons can be reversed in adults. Modulating neuronal $\mathrm{mTOR}$ activity is a potential strategy for axon regeneration after chronic SCI.

Key words: axon regeneration; axon sprouting; chronic spinal cord injury; corticospinal tract; intrinsic axon growth ability; Pten

\section{Significance Statement}

As one of the long descending tracts controlling voluntary movement, the corticospinal tract (CST) plays an important role for functional recovery after spinal cord injury. The regeneration of CST has been a major challenge in the field, especially after chronic injuries. Here we developed a strategy to modulate Pten/mammalian target of rapamycin signaling in adult corticospinal motor neurons in the postinjury paradigm. It not only promoted the sprouting of uninjured CST axons, but also enabled the regeneration of injured axons past the lesion in a mouse model of spinal cord injury, even when treatment was delayed up to 1 year after the original injury. The results considerably extend the window of opportunity for regenerating CST axons severed in spinal cord injuries.

\section{Introduction}

Spinal cord injury (SCI) often causes permanent functional deficits due to the failure of axon regeneration in the adult CNS. This lack of regeneration has been largely attributed to the diminished growth capacity of neurons and to the inhibitory environment (Schwab and Bartholdi, 1996; Filbin, 2006; Harel and Strittmatter, 2006; Zheng et al., 2006; Giger et al., 2010; Liu et al., 2011; Bradke et al., 2012). Many attempts have been made to promote

Received Sept. 1, 2014; revised May 20, 2015; accepted May 27, 2015.

Author contributions: K.L. designed research; K.D., S.Z., Q.Z., S.L., X.G., J.W., and K.L. performed research; J.W. and L.J. contributed unpublished reagents/analytic tools; K.D., S.Z., Q.Z., and K.L. analyzed data; K.L. wrote the paper.

This study was supported in part by the Hong Kong Research Grants Council Theme-Based Research Scheme (Grant T13-607/12R), the National Key Basic Research Program of China (Grant 2013CB530900), the Research Grants Council of Hong Kong Special Administrative Region (Grants AoE/M-09/12, HKUST5/CRF/12R, AoE/M-05/12, C4011-14R, 662011, 662012, 689913, and 16101414), and the Hong Kong Spinal Cord Injury Fund.

*K.D., S.Z., and Q.Z. contributed equally to this work.

The authors declare no competing financial interests.

Correspondence should be addressed to Dr. Kai Liu, Assistant Professor, Division of Life Science, The Hong Kong University of Science and Technology, Clear Water Bay, Kowloon, Hong Kong, People's Republic of China. E-mail: kailiu@ust.hk.

DOI:10.1523/JNEUROSCI.3637-14.2015

Copyright $\odot 2015$ the authors $\quad 0270-6474 / 15 / 359754-10 \$ 15.00 / 0$ axon regeneration in the spinal cord, mostly before or immediately after injury. One of the most convincing pieces of evidence that axons can regenerate across the lesion site after chronic SCI comes from the peripheral dorsal root ganglion (DRG) neurons, which have their central branches in the spinal cord. Applying a conditioning lesion to the peripheral branches of the DRG can boost the growth capacity of chronically injured neurons and encourage regeneration either without gliosis formation (Ylera et al., 2009) or with permissive graft-plus-neurotrophin delivery (Kadoya et al., 2009). Interestingly, ascending axons can actually pass the gliosis-free lesion even in the absence of conditioning lesions, suggesting that these adult peripheral neurons retain a higher axon growth capacity than central neurons (Ylera et al., 2009). However, whether CNS neurons with low intrinsic growth capacity can still regenerate across the injury site in individuals with chronic SCI, in which the inhibitory extracellular matrix at the lesion site is well established, remains an open question.

The loss of the regrowth potential of corticospinal tract (CST) axons is accompanied by a corresponding downregulation of the mammalian target of rapamycin (mTOR) activity in corticospinal motor neurons (CSMNs) upon maturation, which is further reduced after injury (Liu et al., 2010). Deleting Pten, a negative 


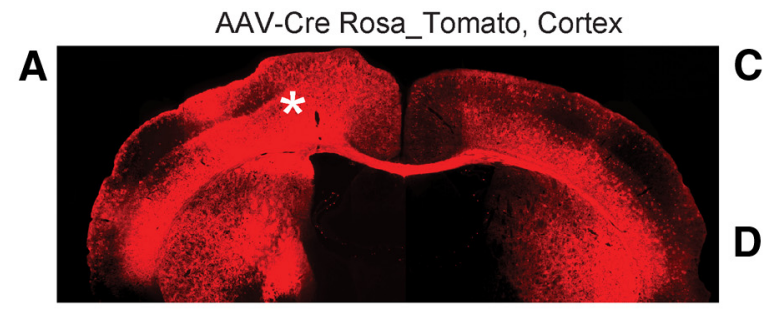

B

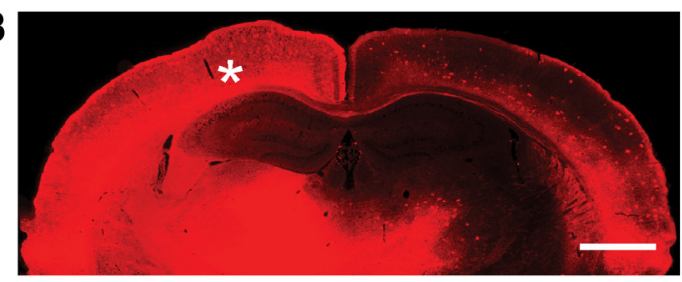

Tomato

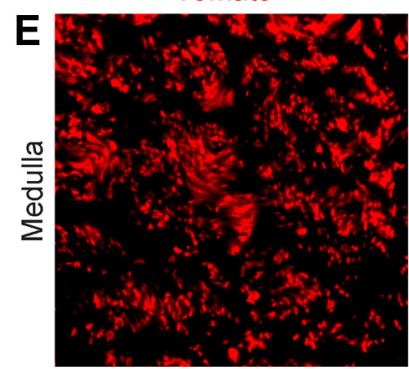

Tomato

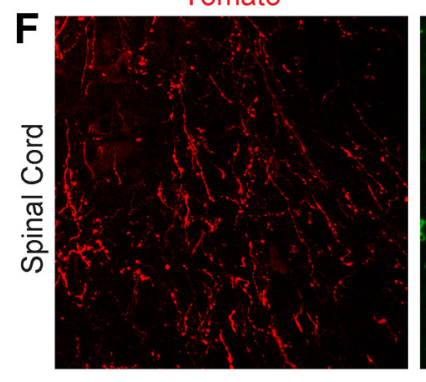

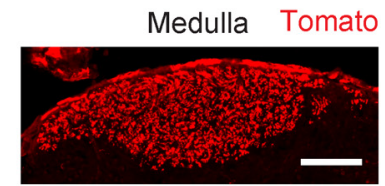

Spinal Cord

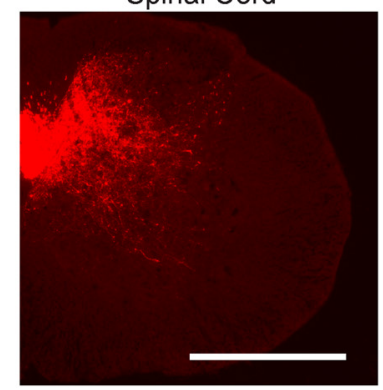

Merge

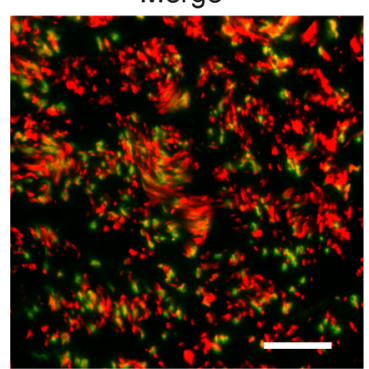

Merge

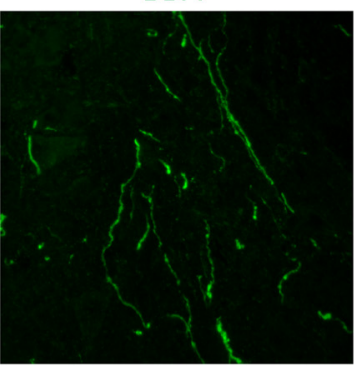

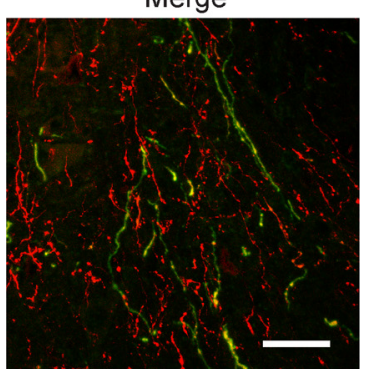

Figure 1. Cortical injection of AAV2/1-Cre results in efficient Cre-dependent Tomato expression in an adult reporter mouse. $\boldsymbol{A}$, $\boldsymbol{B}$, Representative images of the forelimb $(\boldsymbol{A})$ and hindlimb $(\boldsymbol{B})$ regions of the cortex. AAV-Cre was stereotaxically injected into the left side of the sensorimotor cortex of a reporter mouse (Rosa26-I0x-STOP-lox-Tomato) at the age of 8 weeks. The mouse was killed 6 weeks after injection for Tomato protein detection. Asterisks denote the site of AAV injection in the sensorimotor cortex. C, A transverse section of the medulla showing the Tomato ${ }^{+}$CST axons. $\boldsymbol{D}$, A transverse section of the spinal cord showing the Tomato $^{+}$CST axons and their branches. $\boldsymbol{E}, \boldsymbol{F}$, Enlarged regions of a medulla transverse section and a spinal cord transverse section (gray matter) showing the colocalization of Tomato ${ }^{+}$and BDA ${ }^{+}$CST axons. BDA was injected into the cortex at 4 weeks after the virus injection at the same coordinates used for the AAV-Cre injection. Mice were kept for an additional 2 weeks before being killed. Scale bars: $\boldsymbol{A}, \boldsymbol{B}, 1 \mathrm{~mm} ; \boldsymbol{C}, 100 \mu \mathrm{m} ; \boldsymbol{D}, 500 \mu \mathrm{m} ; \boldsymbol{E}, 20 \mu \mathrm{m} ; \boldsymbol{F}, 50 \mu \mathrm{m}$.

regulator of mTOR, in the CSMNs of neonatal mice before injury enhances the compensatory sprouting of uninjured CST axons and enables their regeneration past a spinal cord lesion by preventing the downregulation of mTOR (Liu et al., 2010). Recent studies have indicated that Pten knockdown in the neonatal cortex through shRNA also elicits a growth-enhancing effect (Zukor et al., 2013) and even promotes functional recovery through a combination strategy (Lewandowski and Steward, 2014). However, whether the injury-induced downregulation of the growth capacity can be reversed in adult CSMNs and whether chronically injured CST axons can regenerate are still unexplored. Here we report that Pten deletion could increase collateral sprouting from the intact CST when it commenced 1 week after unilateral pyramidotomy. Initiated either 1 month or 1 year after injury, it enabled the regeneration of CST axons.

\section{Materials and Methods}

Animals and surgeries. All experimental procedures were performed in compliance with animal protocols approved by the Animal and Plant Care Facility at the Hong Kong University of Science and Technology. Rosaloxp-stop-loxp-tdTomato (Rosa_Tomato; catalog \#007905, The Jackson Laboratory), homozygous conditional floxed Pten (Pten ${ }^{f / f}$ ) and $\quad$ Pten $^{f / f} ;$ Rosa-loxp-stop-loxp-tdTomato $\left(\right.$ Pten $^{f / f} ;$ Rosa_Tomato $)$ mice were used in this study. For all surgical procedures, mice were anesthetized with ketamine $(80 \mathrm{mg} / \mathrm{kg})$ and xylazine $(10 \mathrm{mg} / \mathrm{kg})$ through intraperitoneal injection. After the surgery, mice were placed on a warming blanket held at $37^{\circ} \mathrm{C}$ until they were fully awake. After the operation, all mice received subcutaneous injections of meloxicam $(1 \mathrm{mg} / \mathrm{kg})$ as an analgesic.

Pyramidotomy was performed as previously described (Liu et al., 2010) in both female and male mice at 8 weeks of age. The medullary pyramid was unilaterally transected. One week later, the animals underwent cortical adenoassociated virus (AAV) injection. We used selfcomplimentary AAV 2/1 expressing Cre or GFP under a CMV promoter (Vector Biolabs), and the titer was $\sim 10^{13}$ genome copies $/ \mathrm{ml}$. Eight weeks after the AAV injection, the CST was traced with biotin dextran amine (BDA), and mice were kept for an additional 2 weeks. To assess sprouting by BDA tracing, we injected AAVCre or AAV-GFP into Pten ${ }^{\text {flf }}$ mice, with six mice in each group. To assess sprouting by Tomato tracing, we injected AAV-Cre into Rosa_Tomato or Pten ${ }^{\mathrm{ff} f}$;Rosa_Tomato mice, with six mice in each group.

The T8 spinal cord crush was performed as previously described (Liu et al., 2010) in female Pten ${ }^{f / f}$ mice at 8 weeks of age. After T8 laminectomy, the cord was crushed using a pair of customized forceps. Mice with injury were kept for 1 month or 12 months before we injected the AAVs. A total of $2 \mu \mathrm{l}$ of AAV-Cre or AAVGFP was injected into the adult sensorimotor cortex at the following coordinates (in $\mathrm{mm}$ ): anteroposterior/mediolateral: $1.0 / 1.5,0.5 / 1.5$, $-0.5 / 1.5$, and $-1.0 / 1.5$, at a depth of $0.5 \mathrm{~mm}$. Four or 7 months after the AAV injection, the CST was traced with a cortical injection of BDA. In the 1 month delay experiment, we examined the regeneration 4 months after the AAV injection. Eight animals in the AAV-GFP group and 14 animals in the AAV-Cre group were used. Of the 14 mice in the Cre group, 3 mice were injected with AAV-Cre-GFP to facilitate the labeling of CSMNs. We did not find a difference between AAV-Cre-GFP and AAV-Cre, and included all mice for axon quantification. In the 12 month long-term experiments, three animals in the AAV-GFP group and four animals in the AAV-Cre group were used for the 4 month study; and five animals in the AAV-GFP group and six animals in the AAV-Cre group were used for the 7 month study. To label CST axons by anterograde tracing, a total of $2 \mu \mathrm{l}$ of BDA (Life Technologies) was injected into the same sites. Mice were kept for an additional 2 weeks before they were killed to perform histology measurements.

Immunofluorescence staining. Animals were transcardially perfused with $4 \%$ paraformaldehyde. Brains and spinal cords were dissected, postfixed, and then cryoprotected before sectioning at $25 \mu \mathrm{m}$. The pyramidotomy lesion sites were carefully examined, and the completeness of the 

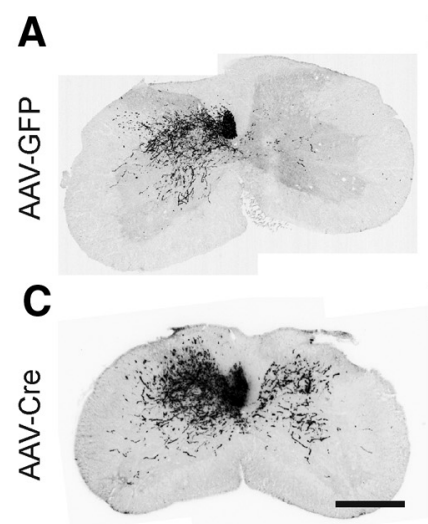

E

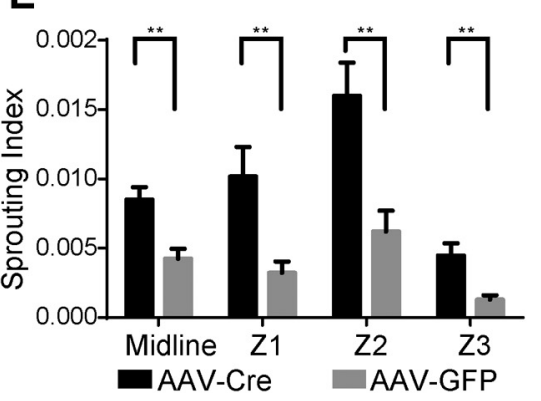

Z1

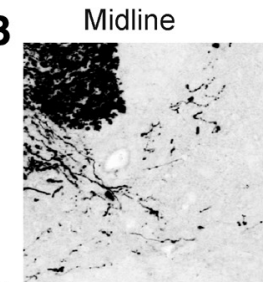

D

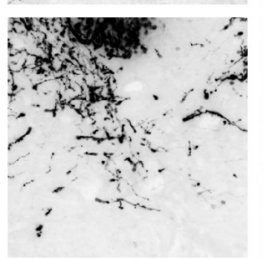

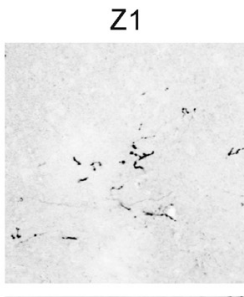

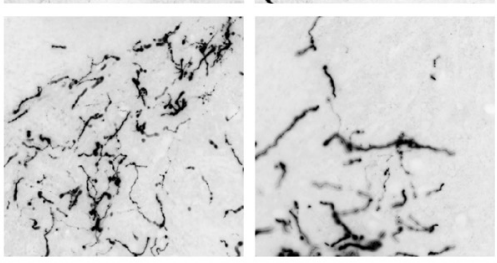

Z2

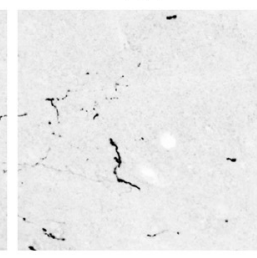

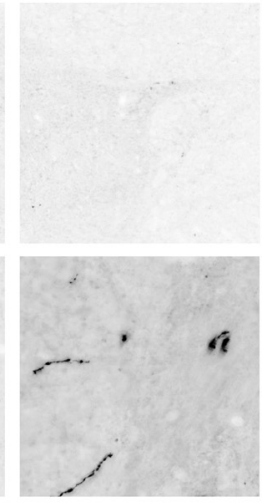

Z3

\section{(1)}
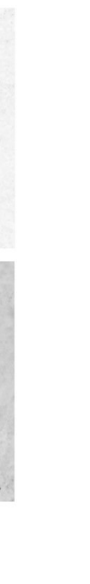

. 

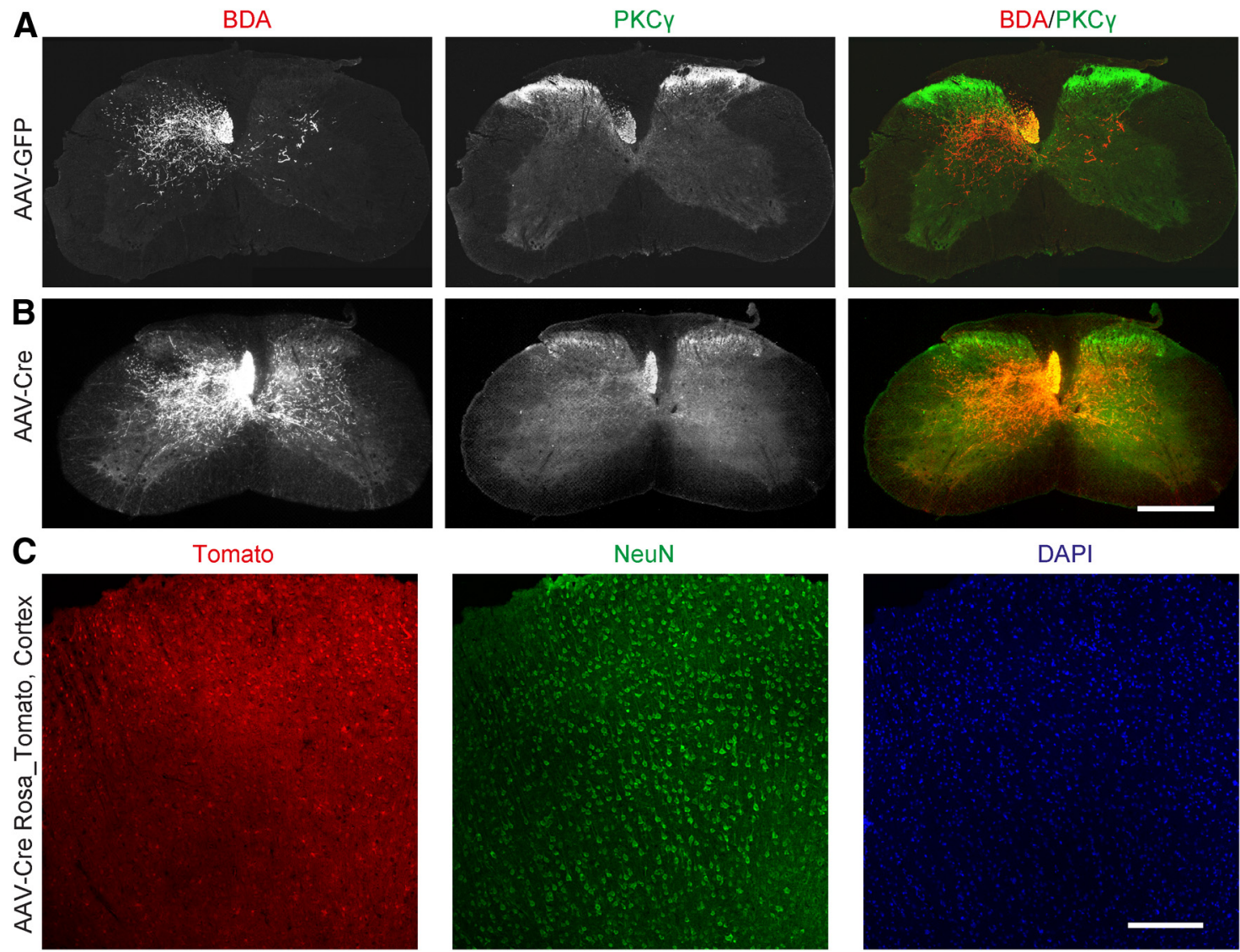

Figure 4. Verification of the completeness of the lesion following unilateral pyramidotomy; AAV1-Cre does not cause neurotoxicity in Rosa_Tomato mice. $A, B$, Representative images of C7 spinal cord transverse sections from adult Pten ${ }^{f / f}$ mice with AAV-GFP or AAV-Cre after unilateral pyramidotomy. Sections were stained with BDA (red) and PKC $\gamma$ antibody (green). Note the unilateral staining of PKC $\gamma$ on the main CST bundle. C, D, Representative images of the cortex with Tomato, NeuN, and DAPI staining around the injection site. We did not observe obvious neuronal death or inflammation. AAV-Cre was injected into the sensorimotor cortex of a reporter mouse (Rosa26-lox-STOP-lox-Tomato) at the age of 8 weeks, and the mouse was killed 6 weeks after the injection. Scale bars: $A, B, 500 \mu \mathrm{m} ; C, D, 200 \mu \mathrm{m}$.

the different markers included the following: p-S6 (catalog \#4858, Cell Signaling Technology); GFAP (catalog \#Z0334, DAKO); red fluorescent protein (catalog \#ab62341, Abcam); GFP (catalog \#A11122, Life Technologies); VGluT1 (catalog \#AB5905, Millipore); Homer1 (catalog \#160004, Synaptic Systems); and PKC $\gamma$ (catalog \#sc-211, Santa Cruz Biotechnology). BDA staining was performed according to the protocol provided for the TSA Cyanine 3 system (Life Technologies). ImageSurfer (Feng et al., 2007) was used to generate 3D reconstructions of the presynaptic and postsynaptic sites, which were triple-labeled with BDA, VGlut1, and Homer1.

Axonal quantifications. Axon numbers were counted manually by researchers who were blinded to the treatment. In the pyramidotomy groups, a horizontal line was first drawn through the central canal and across the lateral rim of the gray matter. Four vertical lines (Mid, Z1, Z2, and Z3) were then drawn to divide the horizontal line into three equal parts. Only axons that intersected with the lines were counted in each section. The results are presented after normalization to the number of CST fibers counted at the level of the medulla.

For the animals with T8 crush injury, the number of fibers caudal to the lesion was analyzed with a fluorescence microscope. The number of intersections between BDA-labeled fibers and a dorsal-ventral line positioned at a defined distance caudal to the lesion center was counted. Every other section of the whole spinal cord was stained. The number of labeled CST axons at different distances from the lesion site was counted in two sagittal sections spanning the level of the dorsal main CST and two additional sections ipsilateral to the main CST.

Statistical analysis. A two-tailed Student's $t$ test was used for the single comparisons between two groups. The rest of the data were analyzed using ANOVA. Post hoc comparisons were performed only when a main effect showed statistical significance. All analyses were conducted through Prism. The data are presented as the mean \pm SEM, and the asterisks indicate statistical significance using the appropriate test.

\section{Results}

Pten deletion in the adult cortex increases CST sprouting after pyramidotomy

Removing Pten to elevate mTOR activity in CSMNs has previously been achieved by injecting AAV-Cre into the neonatal cortex in Pten $^{\text {flf }}$ mice (Liu et al., 2010). However, the widely used AAV serotype $2 / 2$ infects only the adult cortex around the injection track (Hutson et al., 2012). To increase the number of neurons infected in an adult, we used a Cre-expressing, selfcomplementary AAV serotype 2/1 (AAV-Cre) in Pten ${ }^{f / f}$ mice. To validate this strategy, we injected AAV-Cre into the sensorimotor cortex of adult Rosa_Tomato mice and found high Credependent tdTomato protein expression in neurons throughout the sensorimotor cortex at 6 weeks postinjection (Fig. $1 A, B$ ). CST axons were also filled with Tomato and were detected in the medulla (Fig. 1C) and in the spinal cord (Fig. 1D). When injected at the same injection coordinates as the AAV-Cre injection, BDApositive $\left(\mathrm{BDA}^{+}\right)$axons were colabeled with Tomato, as shown in the medulla (Fig. $1 E$ ) and in the gray matter of the spinal cord (Fig. $1 F)$. The mean $\left( \pm\right.$ SEM) number of the Tomato ${ }^{+}$axons was $5778 \pm 160(N=6)$, and the mean number of $\mathrm{BDA}^{+}$axons was $1910 \pm 588(N=6)$. At the level of the medulla, $>92 \%$ of BDA $^{+}$ 

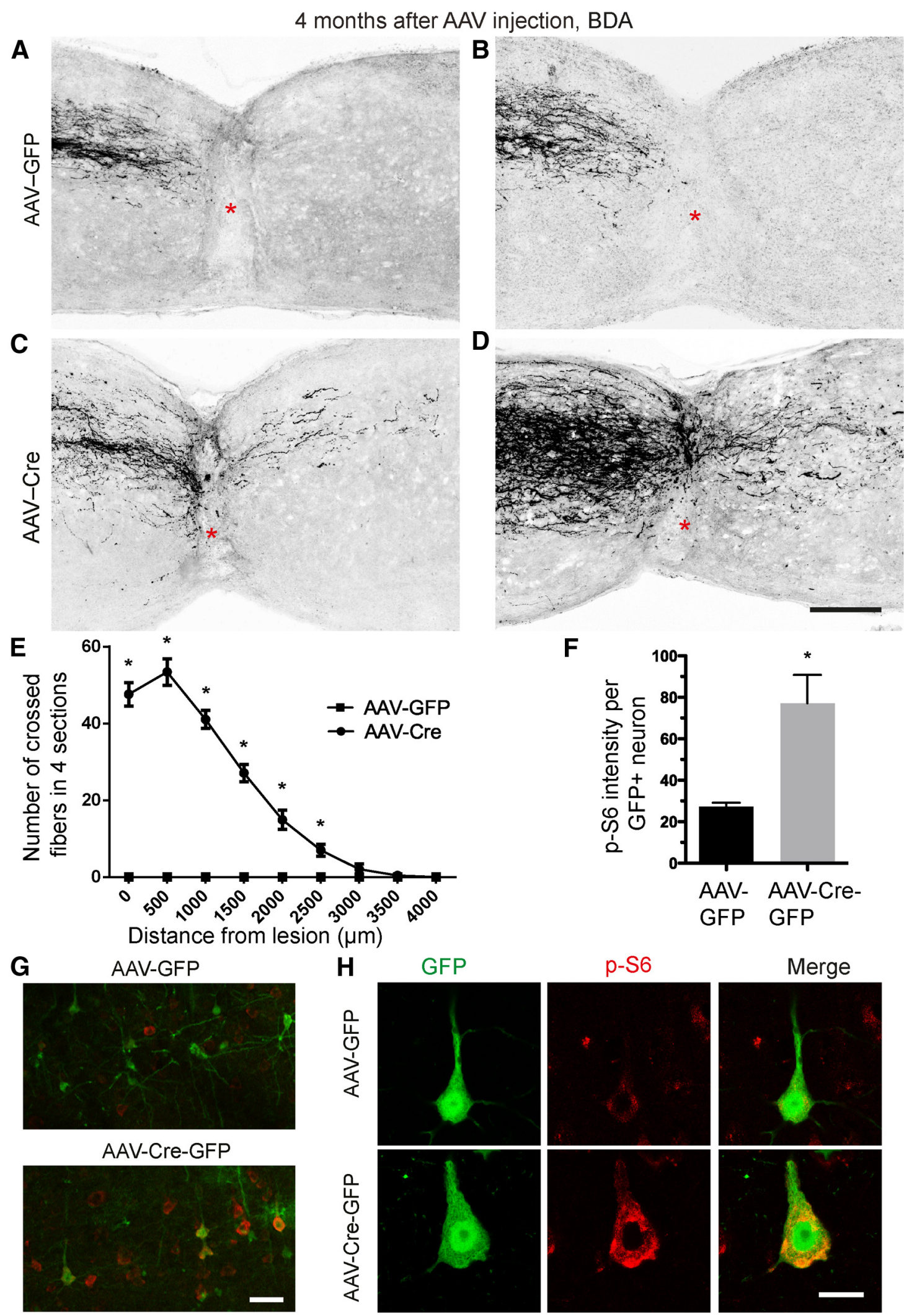

Figure 5. Pten deletion in adult mice promotes CST axon regeneration when AAV is injected 4 weeks after spinal cord crush. The spinal cords of adult Pten ${ }^{\mathrm{f} / \mathrm{f}}$ mice were crushed at T8, and AAV-Cre or AAV-GFP was injected into the hindlimb projection region of the cortex 4 weeks later. Animals survived an additional 4 months, and CST was then traced by BDA.A-D, Representative images from sagittal sections showing the main dorsal CST tract $(\boldsymbol{A}, \boldsymbol{C})$ or collaterals in the gray matter $(\boldsymbol{B}, \boldsymbol{D})$ in Pten ${ }^{\mathrm{f} / \mathrm{f}}$ mice with cortical injection of AAV-GFP $(\boldsymbol{A}, \boldsymbol{B})$ or AAV-Cre $(\boldsymbol{C}, \boldsymbol{D})$. The asterisk denotes the lesion site. Scale bar, $200 \mu \mathrm{m}$. $\boldsymbol{E}$, Quantification of labeled axons in the spinal cord caudal to the lesion site in both groups. ${ }^{*} p<0.05$, ANOVA followed by Fisher's least significant difference test. Eight animals in the AAV-GFP group and 14 animals in the AAV-Cre group were used. Four sections per animal were quantified. $F$, Results of the quantification of the average $p$-S6 intensity of GFP-postive CSMNs in the two groups. The intensity of $>150$ CSMNs from three mice in each group was quantified. ${ }^{*} p<0.05$, Student's $t$ test. $G, \boldsymbol{H}$, Representative images of pS6 and GFP staining in layer 5 of sensorimotor cortex sections from Pten ${ }^{f / f}$ mice with AAV-Cre-GFP or AAV-GFP 4 months after injection in the 1 month delayed SCI model. AAV-Cre was tagged with GFP to show its expression. Scale bars: $\mathbf{G}, 50 \mu \mathrm{m} ; \boldsymbol{H}, 20 \mu \mathrm{m}$. 
A

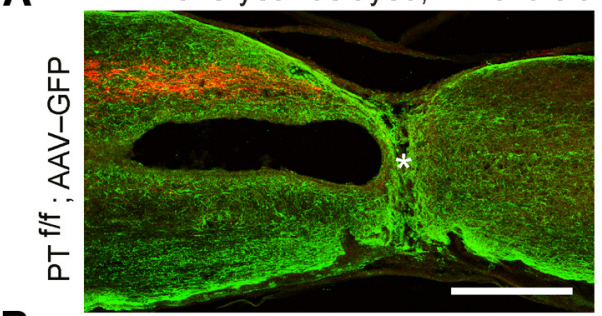

B

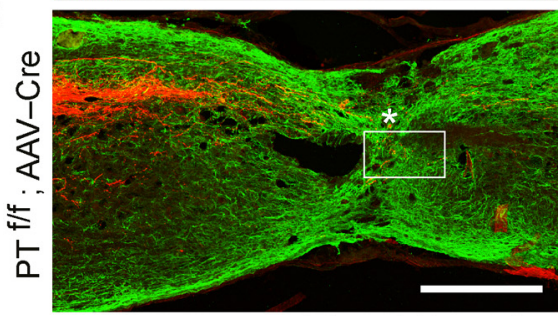

C
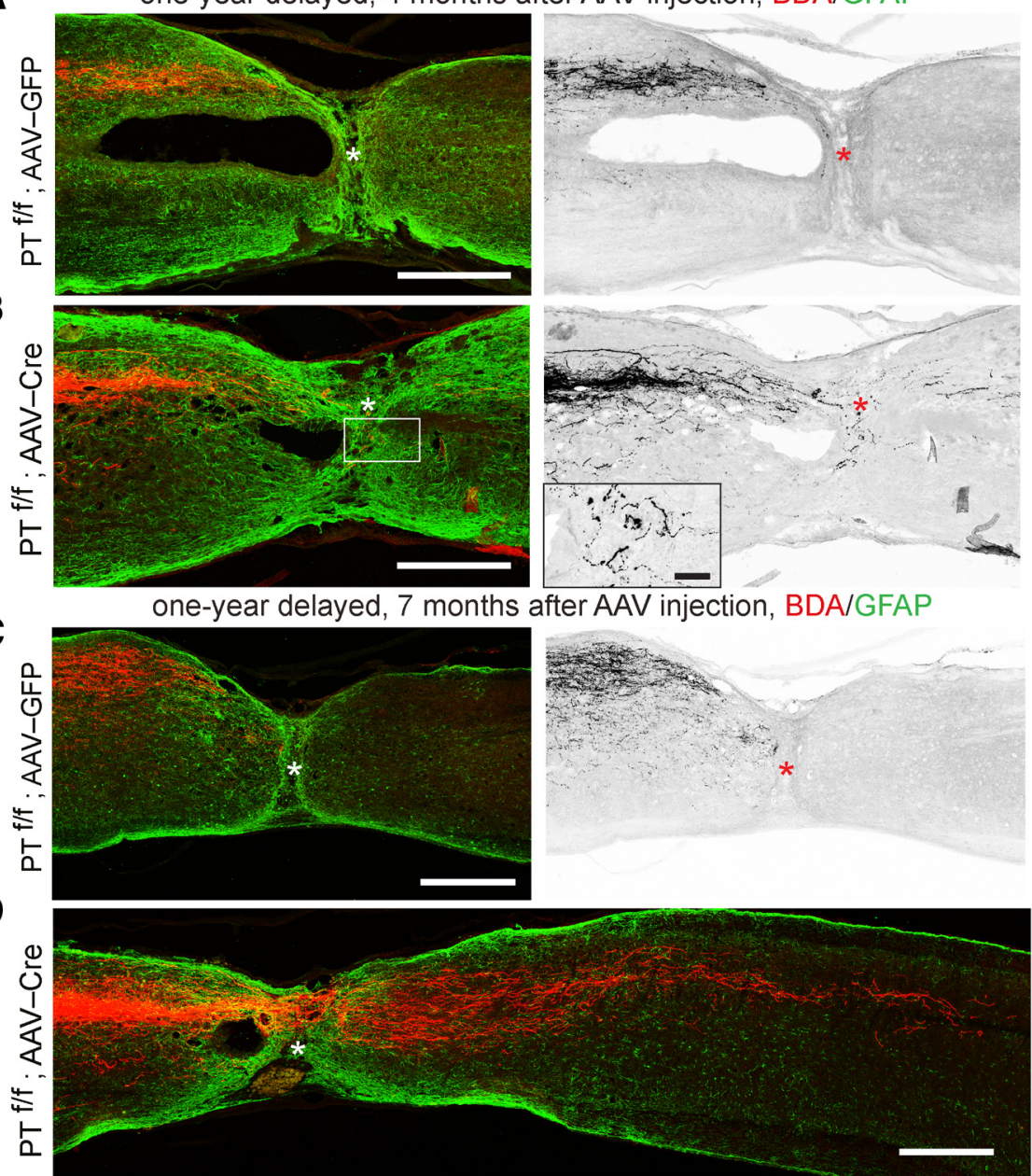

E
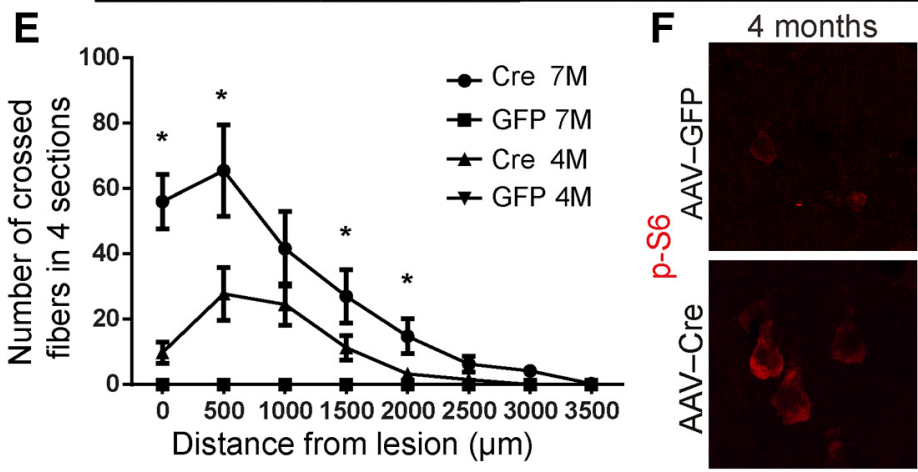

Figure 6. Treatment delayed for 12 months promotes CST axon regeneration in a chronic injury model. The spinal cords of adult Pten ${ }^{f / 4}$ mice were crushed at T8, and AAV-Cre or AAV-GFP was injected into the cortex 12 months later. Animals survived an additional 4 or 7 months before examining the CST axons by BDA.A-D, Representative images from sagittal sections showing the CST axons in $P$ ten ${ }^{f / f}$ mice with cortical injection of AAV-GFP $(\boldsymbol{A}, \boldsymbol{C})$ or AAV-Cre $(\boldsymbol{B}, \boldsymbol{D}) 4$ months $(\boldsymbol{A}, \boldsymbol{B})$ or 7 months $(\boldsymbol{C}, \boldsymbol{D})$ after injection. Left, The sections were costained to detect BDA (red) and GFAP (green). Right, BDA-stained sections were inverted to black and white. The inset shows an enlargement of the boxed region in $\boldsymbol{B}$. The asterisk denotes the lesion site. $\boldsymbol{E}$, Quantification of labeled axons in the spinal cord caudal to the lesion site in both groups of mice 19 months after injury. ${ }^{*} p<0.0167$, ANOVA followed by Bonferroni's post hoc test. Three animals in the AAV-GFP group $(G F P, 4 \mathrm{M}$ ) and four animals in the AAV-Cre group ( $(\mathrm{re}, 4 \mathrm{M})$ were used for the 4 month study; five animals in the AAV-GFP group (GFP, $7 \mathrm{M})$ and six animals in the AAV-Cre group ( $(\mathrm{Cre}, 7 \mathrm{M}$ ) were used for the 7 month study. $F$, Representative images of $\mathrm{p}$-S6 staining in layer 5 of sensorimotor cortex sections from Pten ${ }^{f f / f}$ mice with AAV-Cre-GFP or AAV-GFP 4 or 7 months after injection in the 1 year delayed SCI model. Scale bars: $\boldsymbol{A}-\boldsymbol{D}, 400 \mu \mathrm{m} ; \boldsymbol{B}$, inset, $40 \mu \mathrm{m} ; \boldsymbol{F}, 20 \mu \mathrm{m}$.

axons were also Tomato $^{+}$, and $\sim 35 \%$ of Tomato $^{+}$axons were also traced with BDA. The same injection sites and a several-fold increase in Tomato labeling could explain the colabeling. Thus, using our manipulation, the vast majority of $\mathrm{BDA}^{+}$axons was infected by AAV-Cre, and represented $\mathrm{Cre}^{+}$neurons.
7 months

We next applied a unilateral pyramidotomy in an adult Pten ${ }^{f / f}$ mouse, where CST axons from one side of the sensorimotor cortex were transected above the pyramidal decussation at the level of the medulla. The uninjured side of the cortex was injected with AAV-Cre or AAV-GFP 1 week after the lesioning. The sprouting of intact CST axons into the denervated side of the cervical spinal cord was examined by injecting BDA into the uninjured side of the sensorimotor cortex 8 weeks after the AAV injection. The distribution of labeled axons in the spinal cord was analyzed after an additional 2 weeks. Compared with controls (Fig. 2A,B), Pten deletion (Fig. 2C,D) elicited extensive trans-midline sprouting of adult CST axons from the intact side of the spinal cord into the denervated side (Fig. 2E). To directly demonstrate sprouting by AAVinfected CST axons, we also repeated the pyramidotomy experiment by using Pten $^{\text {flf }}$;Rosa_Tomato and Rosa_Tomato mice. Both groups of mice were injected with AAV-Cre, and we quantified sprouting by normalizing to Tomato ${ }^{+}$axons in the medulla (Fig. $3 A$ ). Consistent with the BDA result, we observed more Tomato ${ }^{+}$ axons sprouting into the denervated contralateral cord after Pten deletion (Fig. $3 B-D)$. Note that the basal level of tracing was substantially increased by using Tomato mice. The completeness of the pyramidotomy was confirmed by PKC $\gamma$ staining (Fig. $4 A, B$ ). NeuN and DAPI staining around the AAV-Cre injection site did not reveal obvious neuronal death (Fig. 4C). Thus, Pten deletion was sufficient to enable these adult neurons to launch a collateral sprouting response after unilateral pyramidotomy.

Pten deletion initiated 4 weeks after T8 crush induces CST regeneration

We then assessed whether Pten deletion after spinal cord injury would reinitiate CST growth. A complete T8 crush that transected all CST axons was performed, and we injected AAV-Cre or AAV-GFP into the right side of the sensorimotor cortex 4 weeks later. The CST was traced 4 months later with BDA. No CST axons extended beyond the lesion site in any of the eight control mice, which is consistent with our previous observations (Liu et al., 2010). We observed the characteristic retraction of CST axons from the injury site. A few fibers could be observed approaching, but rarely within, the lesion site (Fig. 5A,B). When Pten was deleted in CSMNs, we first noticed that the main CST bundle was located closer to the lesion site than in the controls, up to the very edge of the site (Fig. 5C,D). Because all of the mice received crush injuries blindly and because we 
injected AAV 4 weeks after the lesion, when axon retraction had already occurred, it very likely resulted from the enhanced regrowth of injured CST axons. Importantly, 4 months after the AAV-Cre injection, many axons grew into the lesion site and beyond (for some, up to $2.5 \mathrm{~mm}$ ) in all 14 Ptendeleted mice, but not in the 8 controls (Fig. $5 E)$. No labeled axons were detected beyond $4 \mathrm{~mm}$ distal to the lesion sites, which indicates that regeneration was limited and provides further evidence that the lesions were complete. To assess whether Pten deletion elevates neuronal mTOR activity, we examined the p-S6 signal in the cortex. Indeed, compared with the controls, immunostaining for p-S6 was substantially higher in adult Pten-deleted cortical neurons (Fig. $5 F-H$ ). Thus, Pten deletion in the adult cortex promotes the regeneration of CST axons, and this is correlated with reversing the downregulation of $\mathrm{mTOR}$ in injured adult CSMNs.

\section{Pten deletion initiated 12 months after} T8 crush induces CST regeneration

Because a 4 week period of injury may not be considered chronic (Kadoya et al., 2009), we asked whether our findings could be extended to adult CSMNs with chronic injury. A complete T8 crush was performed, and 12 months later we injected AAV-Cre or AAV-GFP into one hemisphere. CST was then traced 4 months later using BDA. In many of the mice from both groups, we noticed a significant enlargement of the central canal rostral to the lesion. The cord around the lesion showed very prominent shrinkage (Fig. 6A,B). In the three controls, a few fibers could rarely be observed approaching the injury site (Fig. 6A). Some of them even turned backward, indicating the inhibitory nature of the lesion boundary. In the four Pten-deleted mice, we did not detect the obvious extension of the main CST bundle. However, a few axons grew into and even beyond the lesion, up to 1.5 $\mathrm{mm}$ (Fig. 6B). Interestingly, some axons were entangled within the lesion site, with abnormal terminals that morphologically resembled retraction bulbs (Fig. 6B, inset). To our surprise, in six mice at 7 months after the AAV-Cre injection, we observed that the main CST bundle extended up to the boundary of the lesion. Numerous axons also regrew into the injury site and across the lesion for up to $3 \mathrm{~mm}$ (Fig. $6 D, E$ ). In the five control mice, this did not occur (Fig. 6C), even at 19 months after injury, which is consistent with the notion that axons in the adult CNS fail to regenerate after injury. Notably, chronically lesioned CSMNs exhibited severe atrophy and diminished mTOR activity (Fig. 6F). A similar atrophy effect has been found in the chronically injured red nucleus neurons (Kwon et al., 2002). In compar-
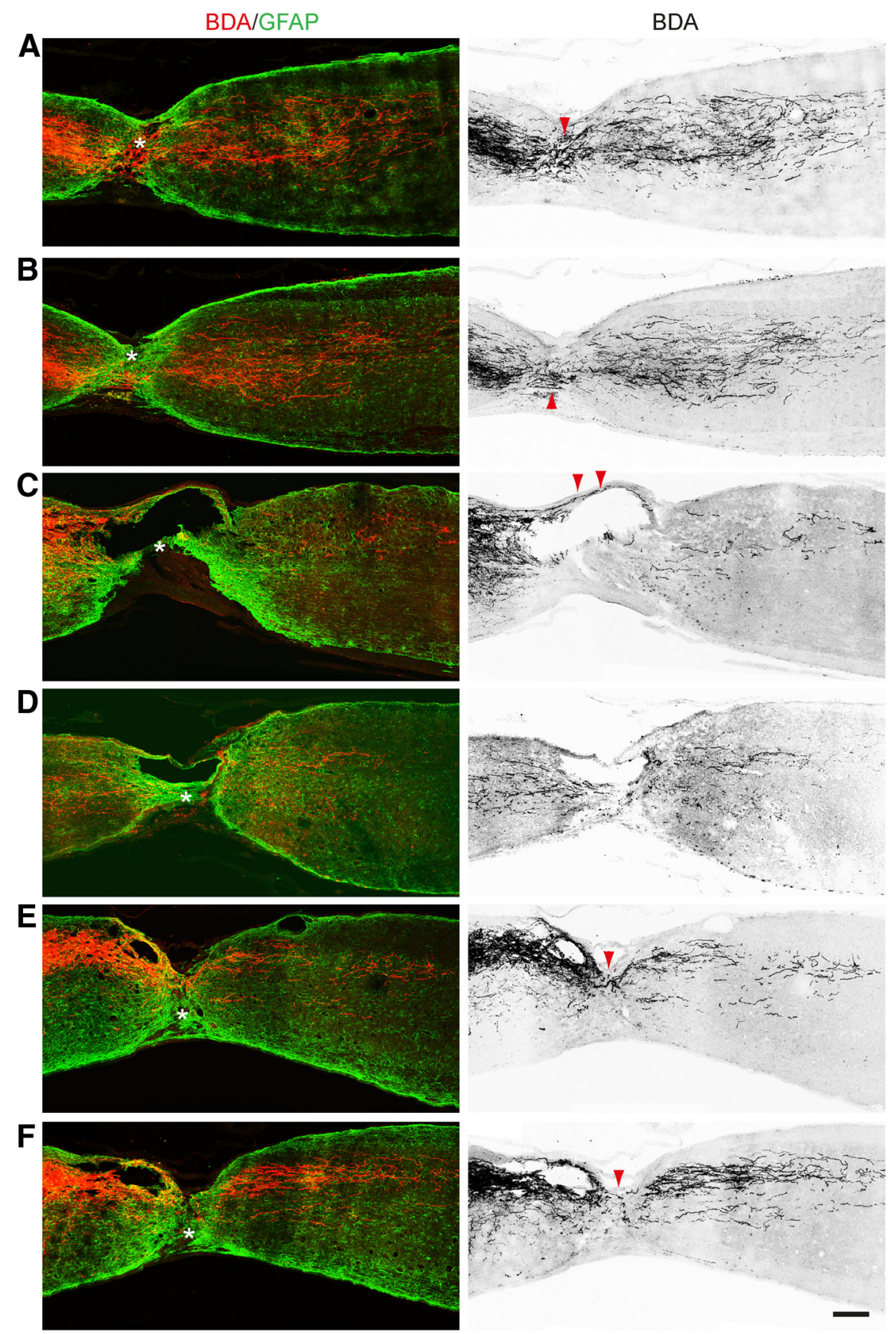

Figure 7. Examples of CST regeneration in the chronic injury model 7 months after AAV-Cre injection. $\boldsymbol{A}-\boldsymbol{F}$, Sagittal sections showing BDA-labeled CST axons in three different Pten $^{\mathrm{f} / \mathrm{f}}$ mice 7 months after cortical injection of AAV-Cre in the 1 year delayed spinal cord injury, with two sections, $\boldsymbol{A}$ and $\boldsymbol{B}, \boldsymbol{C}$, and $\boldsymbol{D}, \boldsymbol{E}$ and $\boldsymbol{F}$, included in each animal. The sections were costained to detect BDA (red) and GFAP (green). Asterisks denote the lesion sites. Red arrows point to the regions in which the axons directly crossed the lesion sites. Most axons were observed on the dorsal side of the lesion $(\boldsymbol{A}, \boldsymbol{C}, \boldsymbol{E}, \boldsymbol{F})$, with some on the ventral side $(\boldsymbol{B})$. Note the large cavity formed in one animal $(\boldsymbol{C}, \boldsymbol{D})$ and a few regenerating axons. Scale bar, $200 \mu \mathrm{m}$.

ison, the p-S6 signal was substantially higher and the cell size much bigger in the cortical neurons of the Pten-deleted adults, in both the 4 month and 7 month groups. Thus, Pten deletion in the adult cortex reverses the downregulation of $\mathrm{mTOR}$ in chronically injured adult CSMNs. Several observations indicate that these axons were regenerated rather than spared. First, many more axons crossed the lesion at 7 months after the AAV injection than crossed at 4 months, suggesting that the axons were continuously growing rather than just being spared. Additional examples from the 7 month group are presented in Figure $7 A-F$. Second, serial 

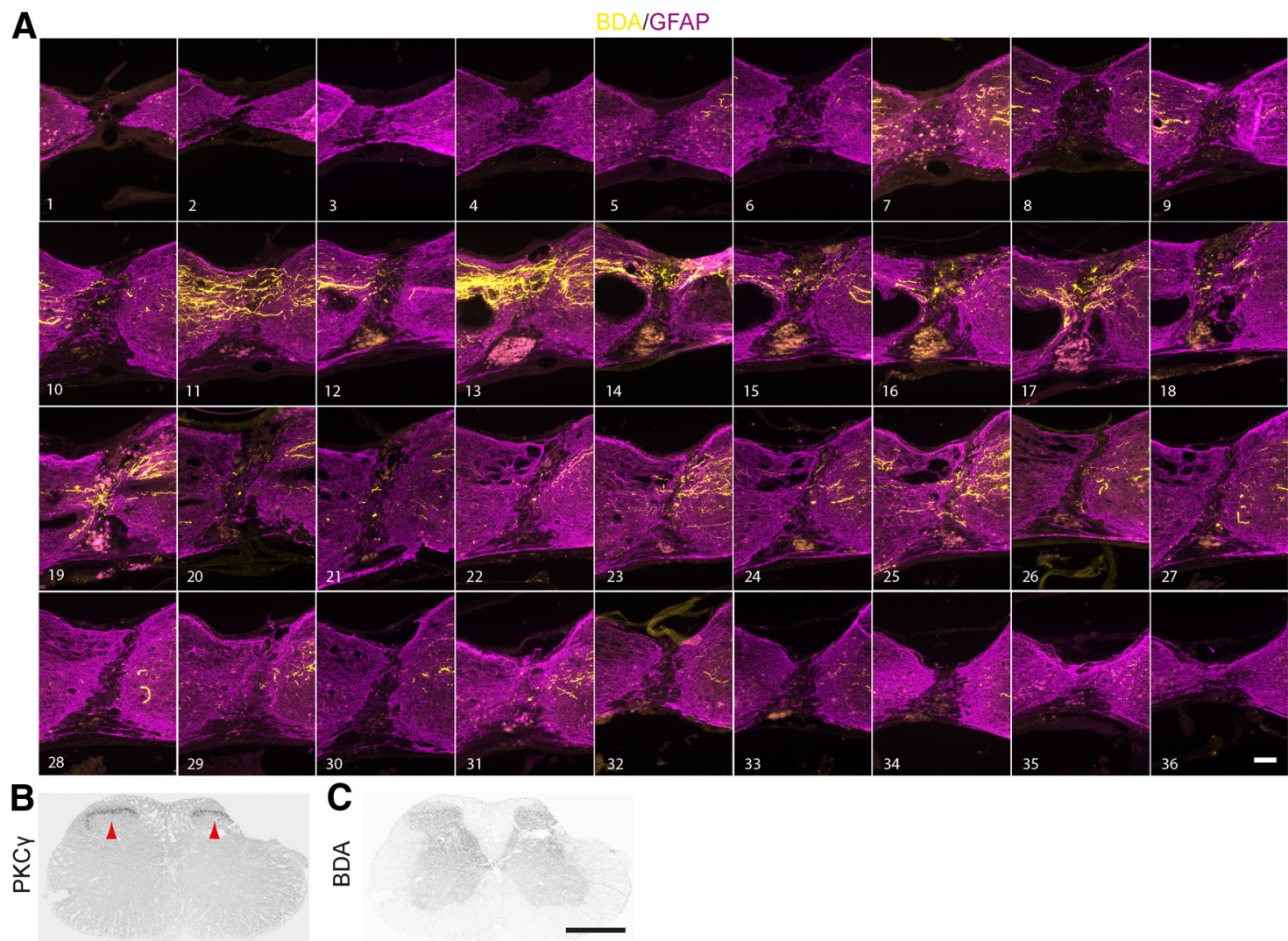

Figure 8. Serial sagittal sections of the spinal cord from a Pten ${ }^{\mathrm{f} / \mathrm{f}}$ mouse 7 months after cortical injection of AAV-Cre in the 1 year delayed model; verification of the completeness of the lesion following the crush. A, Serial sagittal sections of a whole spinal cord with GFAP (magenta) and BDA (yellow) staining, from a Pten ${ }^{\text {f/f }}$ mouse 7 months after cortical injection of AAV-Cre in the 1 year delayed spinal cord injury model, with BDA injected into the right cortex. The midline section through the central canal is marked by number 19 . The majority of the axons appear to pass through the lesion site through discrete areas in the dorsal to middle portions of the lesion (marked by numbers 11,13, 19), whereas a few axons could be traced across the ventral part of the lesion (marked by number 25). Proximal to the lesion, the majority of the CST axons could be observed on the left side of the spinal cord. Distal to the lesion, some CST axons could be observed on both sides of the spinal cord, altering the original projection trajectory. Enlarged images of 13 and 17 are shown in Figures $6 D$, and $9, E$ and $F . B, C$, Representative images of the transverse sections of lumbar spinal cords from adult Pten ${ }^{f / f}$ mice with chronic crush injury. The section was stained with PKC $\gamma$ antibody and BDA. Note that there is PKC $\gamma$ staining in the dorsal horn (red arrowheads) but not in the main CST bundle. Scale bars: $A, 100 \mu \mathrm{m} ; \boldsymbol{B}, C, 500 \mu \mathrm{m}$.

sections of the spinal cord revealed that CST axons past the lesion were not restricted to one side of the distal spinal cord and instead projected bilaterally (Fig. 8A). This is important because normal CST projections are largely unilateral. The presence of substantial numbers of CST axons on the side contralateral to the main tract is strong evidence of regenerative growth and cannot be accounted for by spared axons. Third, in all of the mice with lesions, we found no $\mathrm{BDA}^{+}$axons located $5 \mathrm{~mm}$ distal to the lesion site. We also verified the lesions by the lack of PKC $\gamma$ staining in the main CST bundle of the distal cord (Fig. $8 B, C$ ).

Notably, the majority of regenerating axons projecting into the lesion site were associated with the $\mathrm{GFAP}^{+}$tissue matrix (Fig. $9 A-C, E)$ or even with cells with multipolar or bipolar processes (Fig. 9D). This phenomenon has been consistently observed in previous neonatal Pten deletion and knock-down experiments, and it is likely that $\mathrm{GFAP}^{+}$cells migrated into the lesion sites from both ends of the spinal cord (Liu et al., 2010; Zukor et al., 2013). In the GFAP-negative zone, axons failed to penetrate the lesion site and formed enlarged terminals resembling retraction bulbs (Fig. 9F). The identity and function of these progenitorlike cells need to be further investigated.

We next assessed whether chronically injured corticospinal neurons are able to form synapses. We analyzed samples from Pten $^{\text {f/f }}$ mice with AAV-Cre injection at 12 months after injury. The samples were taken from the gray matter of the spinal cord caudal to the lesion site 19 months after the T8 crush. We assessed whether BDA-labeled regenerating CST axons expressed VGluT1, a presynaptic marker for excitatory synapses, and Homer1, a postsynaptic density protein involved in the targeting of glutamate receptors (Dani et al., 2010). Some BDA-labeled bouton-like structures had VGluT1 staining at the tip of BDAlabeled CST collaterals and along the axonal length (Fig. 10A-C), suggesting a presynaptic terminal. Furthermore, some Homer ${ }^{+}$ patches were at the tips of the bouton-like structures that exhibited BDA and VGlut1 colocalization (Fig. 10D,E), suggesting the accumulation of the molecular machinery characteristic of both presynaptic terminals and postsynaptic sites. These results indicate that regenerating CST axons from Pten-deleted corticospinal neurons have the ability to reform tentative synaptic connections in caudal segments. The functionality of these synapses and the identities of the neurons contacted by the regenerated axons remain unknown.

\section{Discussion}

Together, our results indicate that Pten deletion in postinjured adult corticospinal neurons enables a regenerative response that, to the best of our knowledge, has not been observed previously in the mammalian spinal cord. Both the compensatory sprouting of intact CST axons and the regenerative growth of injured CST axons were significantly increased by Pten deletion. In cortical 
neurons, mTOR activity undergoes a developmental downregulation, and injury further diminishes mTOR activity. Pten deletion in both mature and injured neurons could reverse the mTOR downregulation, and promote the regrowth ability.

Compared with acute injury, axons face more barriers to regenerate after chronic SCI. Axon retraction may further increase the distance that axons need to travel. Extracellular matrices become well consolidated around the chronic lesion site and increase inhibition around the lesion site (Busch and Silver, 2007; Ylera et al., 2009). Neuronal aging may also add obstacles to regrowth (Kang and Lichtman, 2013). In light of all of these challenges, it is indeed surprising to find that CST axons can still regenerate after $>1$ year. This finding indicates that the axon growth machinery can be readily activated, even in older neurons with longterm injury. It also suggests that the injury signal that triggers axon growth is sustained in these mice. It will be interesting to explore this issue further.

Compared with previous results in which Pten was removed or inhibited before the lesion, there could be several possible explanations for why it took substantially longer to observe regeneration in this study. First, in the mice with neonatal Pten deletion, a few injured CST axons reached the lesion edge $10 \mathrm{~d}$ after injury (Liu et al., 2010). It is possible that some "pioneering" axons regrew into the lesion site within a few weeks, before deposition of the inhibitory matrix had been completely established. The initial growth of these axons could have served as a bridge for later axons. This hypothesis is not entirely speculative, as axon bundling across the lesion was observed in this study as well as in previous studies (Neumann et al., 2005; Liu et al., 2010; Zukor et al., 2013). Interestingly, in mice with chronic SCI of 1 year duration, it appears that there is an acceleration of regeneration at 7 months compared with mice at 4 months after AAV-Cre injection. Second, due to retraction or increased inhibition around the lesion, it may take longer for axons to regenerate and reach the lesion site, especially in the mice with chronic SCI. At 4 months after the AAV-Cre injection into mice with chronic injury, the main bundle of CST axons did not reach the lesion boundary. Many more axons reached the lesion edge after an additional 3 months, suggesting the further enhancement of growth over time. Combining this with strategies such as permissive graft bridging ( $\mathrm{Lu}$ et al., 2012) or the removal of inhibitory molecules (Lee et al., 2014; Geoffroy et al., 2015) may further promote axon regeneration after chronic spinal cord injury.

Previously, it has been shown that chronically injured rubrospinal tract axons are capable of regenerating into a peripheral graft (Kwon et al., 2002), and that primary sensory axons can grow across the lesion even at 1 year after injury (Kadoya et al., 2009). Here, we provide evidence that axon regeneration is

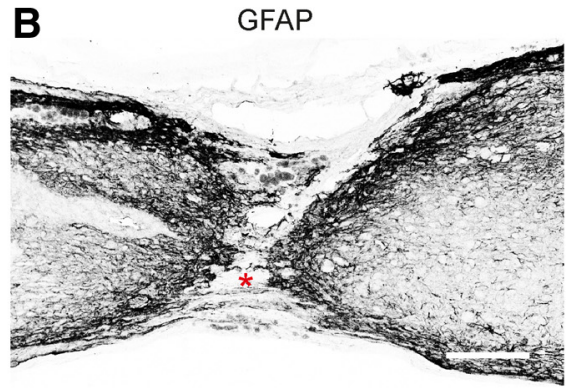

BDA/GFAP

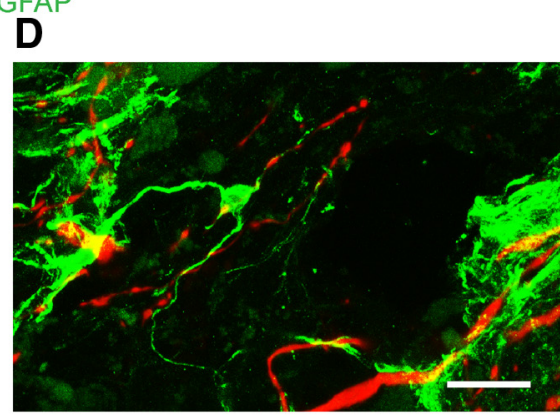

$\mathbf{F}$

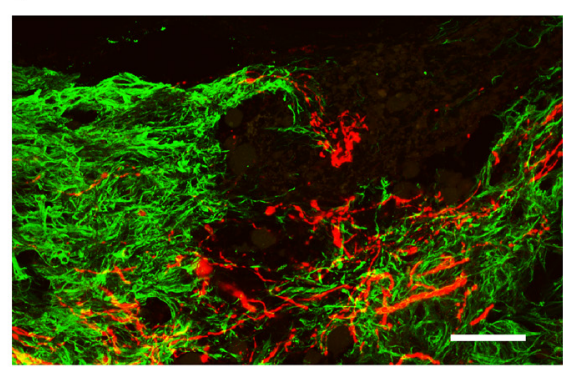

Figure 9. Regenerating axons are associated with $\mathrm{GFAP}^{+}$cells in the lesion site. $\boldsymbol{A}-\boldsymbol{F}$, Sagittal sections showing BDA-labeled CST axons in two Pten ${ }^{f / f}$ mice 7 months after cortical injection of AAV-Cre in the 1 year delayed spinal cord injury model. C, E, Many to an axon turning backward away from the lesion. $\boldsymbol{D}$, A high-magnification image of the boxed area in $\boldsymbol{C}$. BDA-labeled axons are associated with a multipolar GFAP ${ }^{+}$cell within the lesion site. $\boldsymbol{F}, A$ high-magnification image of the boxed area in $\boldsymbol{E}$. Enlarged axon terminals in the GFAP-negative region within the lesion site. Scale bars: $\boldsymbol{A}-\boldsymbol{C}, \boldsymbol{E}, 200 \mu \mathrm{m} ; \boldsymbol{D}, 10 \mu \mathrm{m} ; \boldsymbol{F}, 50 \mu \mathrm{m}$.

achievable in adult corticospinal motor neurons through Pten deletion at 1 year after injury, although the regeneration was mild. It is interesting to find that chronically injured neurons retain the ability to reform tentative synaptic connections. The functional outcome of the regenerated CST axons remains to be tested. Future directions include the development of rational, safe strategies that target the intrinsic neuronal mechanisms through mTOR signaling (Duan et al., 2015).

\section{References}

Bradke F, Fawcett JW, Spira ME (2012) Assembly of a new growth cone after axotomy: the precursor to axon regeneration. Nat Rev Neurosci 13:183193. CrossRef Medline

Busch SA, Silver J (2007) The role of extracellular matrix in CNS regeneration. Curr Opin Neurobiol 17:120-127. CrossRef Medline

Dani A, Huang B, Bergan J, Dulac C, Zhuang X (2010) Superresolution imaging of chemical synapses in the brain. Neuron 68:843-856. CrossRef Medline

Duan X, Qiao M, Bei F, Kim IJ, He Z, Sanes JR (2015) Subtype-specific regeneration of retinal ganglion cells following axotomy: effects of osteopontin and mTOR signaling. Neuron 85:1244-1256. CrossRef Medline

Feng D, Marshburn D, Jen D, Weinberg RJ, Taylor RM 2nd, Burette A (2007) Stepping into the third dimension. J Neurosci 27:12757-12760. CrossRef Medline 
A

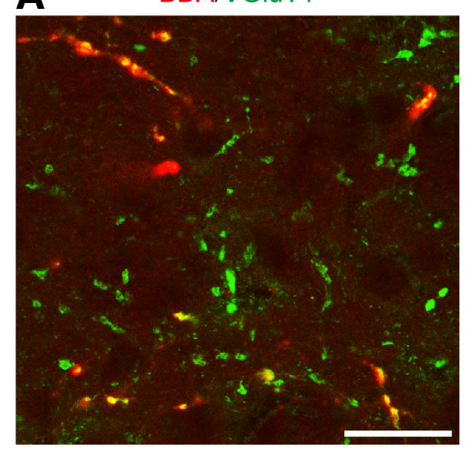

D
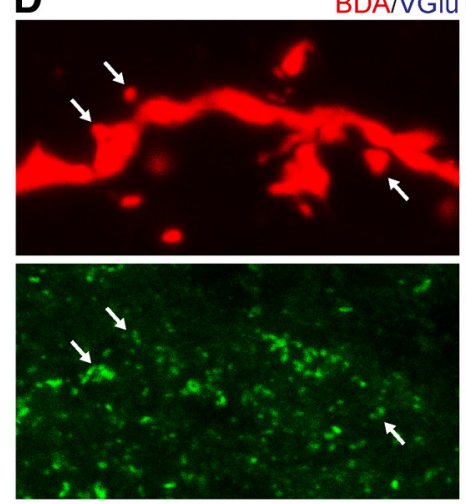

B

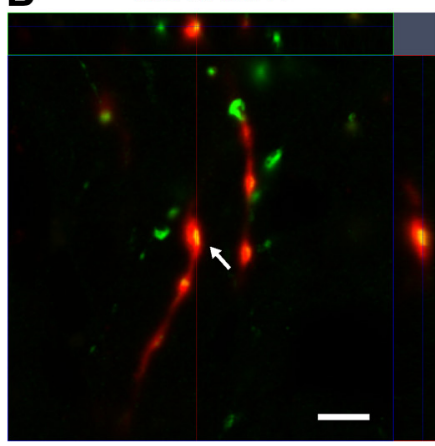

C

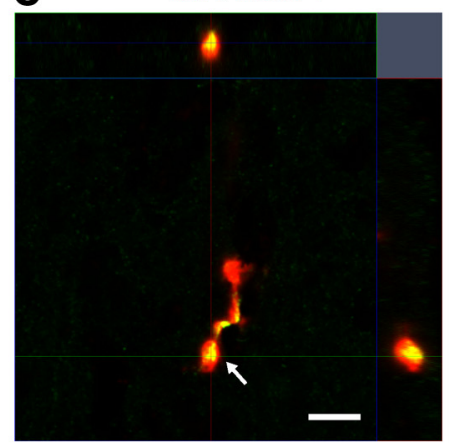

E

BDA/VGluT1/Homer1
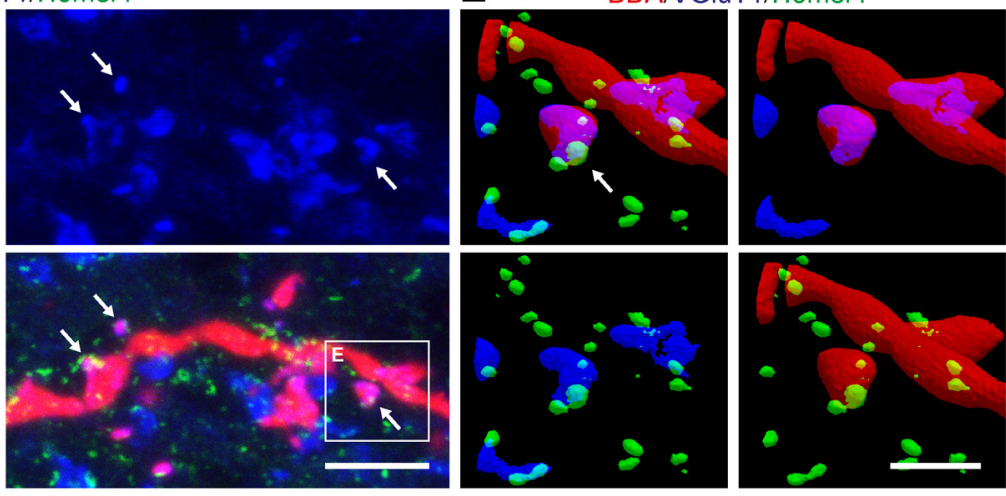

Figure 10. Regenerating CST axons form synaptic structures in the caudal spinal cord after chronic injury. The sections were taken from the distal cord of Pten ${ }^{\mathrm{f} / \mathrm{f}}$ mice $7 \mathrm{months}$ after AAV-Cre injection in the 1 year delayed spinal cord injury model. BDA, VGluT1, and Homer1 signals were used to detect regenerating axons, presynaptic sites, and postsynaptic sites, respectively. $A$, Representative examples of BDA-labeled boutons (red) colocalized with VGluT1 (green) in the gray matter of the spinal cord caudal to the crush site. B, C, Z-stack confocal images with BDA and VGluT1 staining showing the colocalization. Arrows point to the boutons with the centers viewed in three dimensions. $\boldsymbol{D}, \boldsymbol{E}$, Representative examples of BDA-labeled boutons (red) colocalized with VGluT1 (blue) and Homer1 (green) in the gray matter of the spinal cord caudal to the crush site. Arrows point to the triple-labeled synaptic structures formed by the regenerating axons after chronic injury. $\boldsymbol{E}$, Inset from $\boldsymbol{D}$, image of a 3D reconstruction of a BDA-labeled bouton (red) costained with VGluT1 (blue) and Homer1 (green). Scale bars: $\boldsymbol{A}, 20 \mu \mathrm{m} ; \boldsymbol{B}-\boldsymbol{D}, 5 \mu \mathrm{m} ; \boldsymbol{E}, 2 \mu \mathrm{m}$.

Filbin MT (2006) Recapitulate development to promote axonal regeneration: good or bad approach? Philos Trans R Soc Lond 361:1565-1574. CrossRef

Geoffroy CG, Lorenzana AO, Kwan JP, Lin K, Ghassemi O, Ma A, Xu N, Creger D, Liu K, He Z, Zheng B (2015) Effects of PTEN and Nogo codeletion on corticospinal axon sprouting and regeneration in mice. J Neurosci 35:6413-6428. CrossRef Medline

Giger RJ, Hollis ER 2nd, Tuszynski MH (2010) Guidance molecules in axon regeneration. Cold Spring Harb Perspect Biol 2:a001867. CrossRef Medline

Harel NY, Strittmatter SM (2006) Can regenerating axons recapitulate developmental guidance during recovery from spinal cord injury? Nat Rev Neurosci 7:603-616. CrossRef Medline

Hutson TH, Verhaagen J, Yáñez-Muñoz RJ, Moon LD (2012) Corticospinal tract transduction: a comparison of seven adeno-associated viral vector serotypes and a non-integrating lentiviral vector. Gene Ther 19:49-60. CrossRef Medline

Kadoya K, Tsukada S, Lu P, Coppola G, Geschwind D, Filbin MT, Blesch A, Tuszynski MH (2009) Combined intrinsic and extrinsic neuronal mechanisms facilitate bridging axonal regeneration one year after spinal cord injury. Neuron 64:165-172. CrossRef Medline

Kang H, Lichtman JW (2013) Motor axon regeneration and muscle reinnervation in young adult and aged animals. J Neurosci 33:19480-19491. CrossRef Medline

Kwon BK, Liu J, Messerer C, Kobayashi NR, McGraw J, Oschipok L, Tetzlaff W (2002) Survival and regeneration of rubrospinal neurons 1 year after spinal cord injury. Proc Natl Acad Sci U S A 99:3246-3251. CrossRef Medline

Lee DH, Luo X, Yungher BJ, Bray E, Lee JK, Park KK (2014) Mammalian target of rapamycin's distinct roles and effectiveness in promoting compensatory axonal sprouting in the injured CNS. J Neurosci 34:1534715355. CrossRef Medline

Lewandowski G, Steward O (2014) AAVshRNA-mediated suppression of
PTEN in adult rats in combination with salmon fibrin administration enables regenerative growth of corticospinal axons and enhances recovery of voluntary motor function after cervical spinal cord injury. J Neurosci 34:9951-9962. CrossRef Medline

Liu K, Lu Y, Lee JK, Samara R, Willenberg R, Sears-Kraxberger I, Tedeschi A, Park KK, Jin D, Cai B, Xu B, Connolly L, Steward O, Zheng B, He Z (2010) PTEN deletion enhances the regenerative ability of adult corticospinal neurons. Nat Neurosci 13:1075-1081. CrossRef Medline

Liu K, Tedeschi A, Park KK, He Z (2011) Neuronal intrinsic mechanisms of axon regeneration. Ann Rev Neurosci 34:131-152. CrossRef Medline

Lu P, Wang Y, Graham L, McHale K, Gao M, Wu D, Brock J, Blesch A, Rosenzweig ES, Havton LA, Zheng B, Conner JM, Marsala M, Tuszynski MH (2012) Long-distance growth and connectivity of neural stem cells after severe spinal cord injury. Cell 150:1264-1273. CrossRef Medline

Neumann S, Skinner K, Basbaum AI (2005) Sustaining intrinsic growth capacity of adult neurons promotes spinal cord regeneration. Proc Natl Acad Sci U S A 102:16848-16852. CrossRef Medline

Schwab ME, Bartholdi D (1996) Degeneration and regeneration of axons in the lesioned spinal cord. Physiol Rev 76:319-370. Medline

Ylera B, Ertürk A, Hellal F, Nadrigny F, Hurtado A, Tahirovic S, Oudega M, Kirchhoff F, Bradke F (2009) Chronically CNS-injured adult sensory neurons gain regenerative competence upon a lesion of their peripheral axon. Curr Biol 19:930-936. CrossRef Medline

Zheng B, Lee JK, Xie F (2006) Genetic mouse models for studying inhibitors of spinal axon regeneration. Trends Neurosci 29:640-646. CrossRef Medline

Zukor K, Belin S, Wang C, Keelan N, Wang X, He Z (2013) Short hairpin RNA against PTEN enhances regenerative growth of corticospinal tract axons after spinal cord injury. J Neurosci 33:15350-15361. CrossRef Medline 